Theoretical Informatics and Applications

RAIRO: Theoret. Informatics Appl. 38 (2004) 27-48

DOI: $10.1051 /$ ita:2004002

\title{
ASYMPTOTIC BEHAVIOUR OF BI-INFINITE WORDS *
}

\author{
Wit FORYŚ ${ }^{1}$
}

\begin{abstract}
We present a description of asymptotic behaviour of languages of bi-infinite words obtained by iterating morphisms defined on free monoids.
\end{abstract}

Mathematics Subject Classification.68Q.

\section{INTRODUCTION}

Having a morphism $h: A^{*} \rightarrow A^{*}$, and a word $w \in A^{+}$one can consider an infinite iteration $h$ on $w$. Depending on the word and the morphism there are, in general, several possible results of the iteration. We are interested in the case when the result of the iteration process is an infinite word.

The aim of our paper is to describe an asymptotic behaviour of languages of bi-infinite words obtained by iterating morphisms defined on free monoids. The problem was investigated recently by Narbel in [8,9] but imposing on the considered morphisms the restriction that they are expansive. According to the suggestions of Narbel in [9] we extend the considerations for morphisms not necessarily expansive. The obtained results generalize those presented by Narbel in [9] and give a description for both - expansive and non expansive cases. Our results are in the close correspondence with those of Shallit and Wang [10]. However they considered bi-infinite fixed points of morphisms.

Bi-infinite words are very essential in symbolic dynamics giving a description of a motion in the past, future and at the present. Specified families of bi-infinite words substitute subshifts - a symbolic counterparts of discrete dynamical systems. Among various types of subshifts there are subshifts generated by iterating

\footnotetext{
Keywords and phrases. Bi-infinite words, morphisms, iteration, boundary set.

* Results of this paper were presented by the author at Colloquium on Semigroups, Szeged 2000, Hungary.

1 Jagiellonian University, Institute of Computer Science, Nawojki 11, 30-072 Kraków, Poland; e-mail: forysw@ii.uj.edu.pl
} 
a morphism - for example Thue-Morse subshift. This is the main motivation for undertaking the research.

The investigations of asymptotic behaviour of bi-infinite words obtained by iterating morphisms is strongly motivated also within the formal language theory. We refere here to the papers $[1-6,8-10]$.

The paper is organized as follows. In Section 2 some definitions and notions are introduced. In particular we define a metric on bi-infinite words. Within this framework asymptotic properties are studied. In Section 3 we recall some results about iterations of morphisms. Section 4 contains particular results characterizing asymptotic behaviour of bi-infinite words. The main result of the paper, Theorem 5.1, is presented in Section 5.

\section{Notations AND DEFINITIONS}

Let $A$ be any non-empty, finite set called an alphabet. We denote by $A^{*}$ the free monoid generated by $A$ and by $A^{+}=A^{*} \backslash\{1\}$ the free semigroup where $1-$ the empty word stands for identity. For any $X \subset A$ the set of all letters from $X$ that occur in a word $w \in A^{*}$ is denoted by $\operatorname{alph}_{X} w$. A letter from $X$ that occurs in $w$ as the first (counted from the left) is denoted by first ${ }_{X} w$. We need also sometimes the position of the first letter from $X$ in $w$. Let first $X, \mathbb{Z} w$ denote the pair $(a, i)$ where $a=$ first $_{X} w$ and is the $i$-th letter in the word $w$ (counting starts from 0$)$. Dually we introduce $\operatorname{last}_{X} w$ as a letter from $X$ that occurs in $w$ as the last and last $X, \mathbb{Z} w$ as the counterpart of $\operatorname{first}_{X, \mathbb{Z}} w$.

We extend both of these introduced notations for right-infinite and left-infinite words respectively. A word $w \in A^{+}$is primitive if there is no word $v \neq w$ such that $w \in v^{*}$. Recall that two words $v, w \in A^{+}$are conjugates $v \approx w$, if there exist $u_{1}, u_{2} \in A^{*}$ such that $v=u_{1} u_{2}$ and $w=u_{2} u_{1}$. The shift $\sigma:(A \cup 1)^{\mathbb{Z}} \rightarrow(A \cup 1)^{\mathbb{Z}}$ is defined as a function by putting for any $x \in(A \cup 1)^{\mathbb{Z}}, k \in \mathbb{Z}, \sigma(x)(k)=x(k+1)$. In a similar way we define $\sigma^{-1}$ to fulfil the condition $\sigma^{-1}(x)(k)=x(k-1)$. We use in the sequel iterates of $\sigma$ and $\sigma^{-1}$ denoted by $\sigma^{n}$ and $\sigma^{-n}$ (n-fold composition of $\sigma$ and $\sigma^{-1}$ respectively).

Let $\Omega$ denote the set of all functions $w: \mathbb{Z} \rightarrow A \cup\{1\}$ such that if $w(i) \in A$ and $w(j) \in A$ for $i \leq j \in \mathbb{Z}$ then $w(k) \in A$ for $i \leq k \leq j$ and $0 \in \operatorname{supp} w$ where supp $w=w^{-1}(\bar{A})$. Any element of $\Omega$ is called a word. From the definition it follows that $\operatorname{supp} w$ is a discrete interval for any word $w$. A restriction of a word $w$ to a discrete interval included in $\mathbb{Z}$ is called a factor of $w$ and denoted by $w[i, j], w(-\infty, i)$ etc. according to the form of the interval. Note that a factor is not necessarily a word but is of course a partial function defined on $\mathbb{Z}$. We say that a word $w \in \Omega$ is factorized in factors $u_{1}, v, u_{2}$ and denote it by $w=u_{1} v u_{2}$ if there exist $k, l \in \mathbb{Z}$ and $k \leq l$ such that

$$
u_{1}=w(-\infty, k], \quad v=w(k, l], \quad u_{2}=w(l,+\infty) .
$$

The case $k=l$ in the above definition means that the factorization is of the form $w=u_{1} u_{2}$. 
Any factor $x$ of a word $w$ can be extended to a function defined on $\mathbb{Z}$ putting for all integers outside the factor's domain the value 1 . Shifting the obtained function by $\sigma^{k}$ for a suitable $k$ we obtain a word $\bar{x} \in \Omega$. Hence we say that the factor $x$ defines a word $\bar{x}$ (in fact an equivalence class). Again for simplicity we denote a word defined by a factor $x$ by the same symbol $x$. We will use in the sequel properties of words defined by factors as properties of the factors.

In this framework one can consider any finite non-empty word $w$ in $A^{*}$ as a function such that $\operatorname{supp} w$ is a finite interval containing 0 . In the case when $\operatorname{supp} w=[0,|w|-1]$ we say that it is the normal form of a finite word $w$. In a similar way right-infinite and left-infinite words $w$ can be considered and their normal forms introduced having $\operatorname{supp} w$ equal to $[0,+\infty)$ or $(-\infty, 0]$ respectively. Hence we can consider $\Omega$ as the set of words - finite, one-sided infinite and biinfinite. For a word $w \in \Omega$ the value $w(0)$ is called the origin of $w$.

Finally, for any $w$ in $\Omega$ we introduce the notation $\sigma^{*}(w)$ for the set $\sigma^{*}(w)=$ $\left\{\sigma^{k}(w) \in \Omega: k \in \mathbb{Z}\right\}$ and extend it to any language $L \subset A^{*}$ as follows

$$
\sigma^{*}(L)=\bigcup_{w \in L} \sigma^{*}(w)
$$

We interpret all sequences in $\sigma^{*}(w)$ as a symbolic description of the same dynamics. They differ only in the point of the observation has started (the origin). From this point of view it is obvious to identify all words in $\sigma^{*}(w)$. More formally one can consider the quotient space $\Omega / \sigma^{*}$ where two bi-infinite words $u, w$ are equivalent if and only if $\sigma^{*}(u)=\sigma^{*}(w)$. In this framework finite words and one-sided infinite words are embedded into $\Omega / \sigma^{*}$.

To avoid notational complications and to make all our results more readable we use a phrase "a word $w$ " in all cases where the position of the origin is unessential. Hence in all these cases one can consider $w$ as an equivalence class in $\Omega / \sigma^{*}$. We will use a phrase "equal modulo the origin" when we would like to stress the fact that words are functions in $\Omega$ and they are in the same equivalence class. In all cases when origins are essential (for example considering convergency) the origins of words will be explicitly pointed out.

In the definitions of a metric that we introduce now the essential role is played by the origin of considered words. Define the metric on $\Omega$ by putting for any $w \neq v$ in $\Omega, d(w, v)=2^{-(k+1)}$ where $k$ is the maximal nonnegative integer such that $w[-k, k]=v[-k, k]$ if $k$ exists. In the opposite case put $k=-1$. In case $w=v$ put $d(w, v)=0$. Note that for words $w, v$ which have only the same origin that is $w(0)=v(0)$ we have $d(w, v)=\frac{1}{2}$.

Let $h: A^{*} \rightarrow A^{*}$ be a morphism of a free monoid $A^{*}$. We will extend $h$ to $\Omega$ and then to $\Omega / \sigma^{*}$. We will use in the sequel so called pointed words, that is, words in $\Omega$ and unpointed words, that is, equivalence classes in $\Omega / \sigma^{*}$. It enforces us to define an extension of a morphism on $\Omega$ which fulfils morphism conditions and transforms in some way the origin of a word.

Let a finite nonempty word $w$ be in the normal form. Hence $\operatorname{supp} w=[0, k]$ for some $k \in \mathbb{N}$. For $i=0, \ldots, k w^{(i)}$ is the only word in $[w] \in \Omega / \sigma^{*}$ such that 
$w^{(i)}=\sigma^{i} \circ w$. It means that $w^{(i)}$ fixes the origin on the $i$-th letter of the finite word $w$. For a bi-infinite word $w=\ldots a_{-1} a a_{1} \ldots$ for which $w(0)=a$ let us fix $i \in \mathbb{N}$ such that $0 \leq i \leq|h(a)|-1$. Define

$$
h^{(i)}(w)=h^{(i)}\left(\ldots a_{-1} a a_{1} \ldots\right)=\ldots h\left(a_{-1}\right) h^{(i)}(a) h\left(a_{1}\right) \ldots
$$

where $h^{(i)}(a)=h(a)^{(i)}$. Hence $h^{(i)}$ fixes the origin on the $i$-th letter of the word $h(a)$ considered in the normal form.

Observe that the introduced definition of $h^{(i)}$ agrees with the definition of the infinite iteration of $h$ of Shallit and Wang [10]. Namely, let $h(a)=w=v_{L} a v_{R}$ for some $v_{L}, v_{R} \in A^{*}, a \in A$ and consider a word $h(a)$ in the normal form. Let $\left|v_{L} a\right|=i$. In [10] an infinite iteration of $h$ on the letter $a$, depending on $i$ is defined as the bi-infinite word having the following factorization

$$
h^{*, i}(a)=\ldots h^{2}\left(v_{L}\right) h\left(v_{L}\right) v_{L} a v_{R} h\left(v_{R}\right) h^{2}\left(v_{R}\right) \ldots
$$

and such that $h^{*, i}(a)(-\infty, 0]=\ldots h^{2}\left(v_{L}\right) h\left(v_{L}\right) v_{L} a$.

The integer $i$ fixes the origin in $h^{*, i}(a)$ by pointing out the origin in the subword $v_{L} a v_{R}$.

It is easy to observe that in the introduced framework the sequence of finite iterations of $h^{(i)}(a)$ denoted by $h^{n, i}(a)$ converges to $h^{*, i}(a)$ if $n$ grows to infinity. Hence the infinite iteration of $h$ in the above sense is denoted in the sequel by $h^{*, i}(a)$ for a suitable $i$ and a letter $a$.

A bi-infinite word $w$ is a fixed point of a morphism $h$ if the words $w$ and $h(w)$ are equal modulo the origin. We denote this fact by $h(w)=w$.

Our research is focused on the set of all finite iterations of a morphism on letters from the alphabet. We consider these iterations as pointed words because we are interested in the asymptotic behaviour of iterations. This set is denoted by $L_{h}$ for a fixed morphism $h$ and is defined as

$$
L_{h}=\bigcup_{n \in \mathbb{N}, a \in A}\left[h^{n}(a)\right]
$$

where $\left[h^{n}(a)\right]$ denotes the equivalence class of a finite word $h^{n}(a)$. Equivalently $L_{h}=\sigma^{*}\left\{h^{n}(a) \in \Omega: a \in A, n \in \mathbb{N}\right\}$ where $h^{n}(a)$ denotes the finite word $h^{n}(a)$ in the normal form.

Considering the asymptotic behaviour of the set $L_{h}$ we define the boundary set of $L_{h}$ denoted by $\partial L_{h}$ as the set of all words $w \in \overline{L_{h}} \backslash L_{h}$ where $\overline{L_{h}}$ denotes the closure of $L_{h}$ in the metric space $(\Omega, d)$. 
In the sequel we use the following notations according to Head [5]:

$$
\begin{aligned}
M & =\left\{a \in A: \exists r \in \mathbb{N} \backslash\{0\}, h^{r}(a)=1\right\} ; \\
M_{R} & =\left\{a \in A: \exists r \in \mathbb{N} \backslash\{0\}, h^{r}(a) \in M^{*} a M^{*}\right\} ; \\
I_{M_{R}} & =\left\{a \in A \backslash M_{R}: \exists r \in \mathbb{N} \backslash\{0\}, h^{r}(a) \in\left(M_{R} \cup M\right)^{+} \backslash M^{*}\right\} ; \\
E & =\left\{a \in A: \exists r \in \mathbb{N} \backslash\{0\}, h^{r}(a)=w_{L} a w_{R}, w_{L} w_{R} \in\left(A^{+} \backslash M^{*}\right)\right\} ; \\
I_{E} & \left.=\{a \in A \backslash E: \exists r \in \mathbb{N} \backslash 0\}, h^{r}(a) \in A^{*} E A^{*}\right\} .
\end{aligned}
$$

Letters in $M$ are called mortal, in $M_{R}$ monorecursive and in $E$ expansive. Letters in $I_{M_{R}}$ or $I_{E}$ lead to a monorecursive or expansive letter, respectively.

Note that for any letter $a \in M_{R} \cup E$ there exists the smallest positive integer $N(a)$ such that $h^{N(a)}(a) \in A^{*} a A^{*}$.

We will illustrate the introduced notions, as well as the obtained in the sequel results considering all along the paper a morphism $h$ defined below.

Example 2.1. Let $A=\left\{a, b, c, d, e, a^{\prime}, b^{\prime}, c^{\prime}\right\}$ be an alphabet. Let $h$ be defined as follows

$$
\begin{array}{cc}
a \rightarrow b e & a^{\prime} \rightarrow e b^{\prime} \\
b \rightarrow a e & b^{\prime} \rightarrow e a^{\prime} \\
c \rightarrow a e a^{\prime} & c^{\prime} \rightarrow a^{\prime} e a \\
d \rightarrow \text { aea } & e \rightarrow e .
\end{array}
$$

We have: $M=\emptyset, M_{R}=\{e\}, I_{M_{R}}=\emptyset, E=\left\{a, b, a^{\prime}, b^{\prime}\right\}, I_{E}=\left\{c, c^{\prime}, d\right\}$. If for the use of this example only we add to the alphabet $A$ two letters $f, g$ and extend the morphism $h$ putting $h(f)=e g$ and $h(g)=1$ then $M=\{g\}$ and $I_{M_{R}}=\{f\}$.

A word $w \in \partial L_{h}$ is said to be a pasted word (of left-infinite and right-infinite words), if there exist

(i) $v_{L} \in \partial L_{h}$ left-infinite, $v_{R} \in \partial L_{h}$ right-infinite,

(ii) a finite word $m \in\left(M \cup M_{R}\right)^{*}$ such that $w=v_{L} m v_{R}$ modulo the origin.

Let $\delta L_{h}$ denote the set of all words $w \in \partial L_{h}$ which are the pasted words or left or right infinite words. The set $\delta L_{h}$ was characterized in [9] by Narbel assuming that the considered morphisms are expansive. Just from the definition the set of pasted words $\delta L_{h}$ is included into the boundary set $\partial L_{h}$. Presented below example shows that, in general, it is a strict inclusion. Words of $\partial L_{h}$ that remain outside the set $\delta L_{h}$ are the subject of consideration in Matyja [7].

Example 2.2. Add a letter $f$ to the alphabet $A$ and extend again for the use of this example only the morphism $h$ putting $h(f)=e f e$. Now we have ${ }^{\omega} e f e^{\omega} \in \partial L_{h}$ and $e^{\omega} \in \partial L_{h}$ as the limit of the sequence $h^{2 n}\left(a^{\prime}\right)=e^{2 n} a^{\prime}$ for $n \in \mathbb{N}$. Similarly $\omega_{e} \in \partial L_{h}$. However ${ }^{\omega} e f e^{\omega} \notin \delta L_{h}$ as $f \in E$.

The aim of the paper is to establish a description and properties of the words in $\delta L_{h}$ by means of the iterations of the morphism $h$ and fixed point property. 


\section{Preliminary Results}

In this section we recall some basic facts about morphism iteration and words which are obtained during this operation. We start with two lemmas which summarize some results within combinatorics on words obtained by Head [4], Head and Lando [5] and Foryś and Head [2]. The proofs are omitted and any interested reader is requested to consult the references. This part presents results of finite words only. One can consider the words as equivalence classes or as finite sequences of letters (normal forms). In the same manner morphism iterations can be considered.

Lemma $3.1[4,5]$. The sets $M, M_{R}, I_{M_{R}}, E, I_{E}$, are pairwise disjoint and effectively constructable.

Let us denote $M_{F}=M \cup M_{R} \cup I_{M_{R}}$ for a fixed morphism $h: A^{*} \rightarrow A^{*}$. For any $a \in E$ there exists the smallest integer $r_{a}$ such that the condition (i) $h^{r_{a}}(a) \in\left(M_{F}^{+} \backslash M^{*}\right) a A^{*}$ is fulfiled.

Hence we obtain a partition of $E$. Any block in this partition, denoted by $R_{M}^{r}$ consists of all letters $a \in E$ that have the same common minimal exponent $r$ for the condition (i). During the iteration of $h$ if a letter $a \in R_{M}^{r}$ occurs as the first letter from $E$ on some stage of the iteration process then after every $r$ iterations the configuration repeats. We define in the same manner and for the same reasons subsequent partitions of $E$ changing the condition (i) respectively to:

(ii) $h^{r_{a}}(a) \in M^{*} a\left(A^{+} \backslash M^{*}\right)$;

(iii) $h^{r_{a}}(a) \in A^{*} a\left(M_{F}^{+} \backslash M^{*}\right)$;

(iv) $h^{r_{a}}(a) \in\left(A^{+} \backslash M^{*}\right) a M^{*}$.

The blocks of the defined partitions are denoted by $R^{r}, L_{M}^{r}$ and $L^{r}$ respectively.

Taking into account only non-empty of the introduced above sets we define

$$
\begin{aligned}
I_{R_{M}}^{r} & =\left\{a \in\left(E \cup I_{E}\right) \backslash R_{M}^{r}: \exists s \in \mathbb{N}, h^{s}(a) \in M_{F}^{*} R_{M}^{r} A^{*}\right\}, \\
I_{R}^{r} & =\left\{a \in\left(E \cup I_{E}\right) \backslash R^{r}: \exists s \in \mathbb{N}, h^{s}(a) \in M_{F}^{*} R^{r} A^{*}\right\}, \\
I_{L_{M}}^{r} & =\left\{a \in\left(E \cup I_{E}\right) \backslash L_{M}^{r}: \exists s \in \mathbb{N}, h^{s}(a) \in A^{*} L_{M}^{r} M_{F}^{*}\right\}, \\
I_{L}^{r} & =\left\{a \in\left(E \cup I_{E}\right) \backslash L^{r}: \exists s \in \mathbb{N}, h^{s}(a) \in A^{*} L^{r} M_{F}^{*}\right\} .
\end{aligned}
$$

Letters in $I_{R_{M}}^{r}$ are exactly those which are outside $R_{M}^{r}$ but lead to a letter in $R_{M}$. Iterating $h$ on a letter from $I_{R_{M}}^{r}$ we obtain after $s$ iterations a letter $a \in R_{M}^{r}$ - the first letter from $E$ that occurs in this iteration. And after every $r$ iterations of $h$ the configuration repeats. The similar meaning is for $I_{R}^{r}, I_{L_{M}}^{r}$ and $I_{L}^{r}$.

Example 3.2. Continuing considerations of the morphism $h$ defined in Example 2.1 we have $M_{F}=\{e\}$. For $r=2$ we obtain $L_{M}^{2}=R^{2}=\{a, b\}, R_{M}^{2}=L^{2}=$ $\left\{a^{\prime}, b^{\prime}\right\}$ and finally $I_{R_{M}}^{2}=\left\{c^{\prime}\right\}, I_{R}^{2}=\{c, d\}, I_{L_{M}}^{2}=\left\{c^{\prime}, d\right\}, I_{L}^{2}=\{c\}$.

Lemma 3.3 [5]. The defined sets $R_{M}^{r}, I_{R_{M}}^{r}, R^{r}, I_{R}^{r}, L_{M}^{r}, I_{L_{M}}^{r}, L^{r}, I_{L}^{r}$, are effectively constructable for any $r \in[1, \sharp A]$. If $r>\sharp A$ then all the sets are empty. 
For a word $w \in M_{F}^{*}$ we have $h^{\sharp A}(w) \in\left(M \cup M_{R}\right)^{*}$ and there are two possibilities:

1. $\operatorname{alph}_{M_{R}}\left(h^{\sharp A}(w)\right) \neq \emptyset$. Thus $\operatorname{alph}_{M_{R}}\left(h^{\sharp A}(w)\right)=\left\{a_{1}, \ldots, a_{p}\right\}$ and for every $i \in\{1, \ldots, p\}$ there exists a least integer $r_{i} \in[1, \sharp A]$ such that $h^{r_{i}}\left(a_{i}\right) \in$ $M^{*} a_{i} M^{*}$. In this case we denote l.m. $(w)=$ l.c.m. $\left\{r_{1}, \ldots, r_{p}\right\}$ and call this number the least common multiple of the word $w$;

2. $\operatorname{alph}_{M_{R}}\left(h^{\sharp A}(w)\right)=\emptyset$. In this case we put l.m. $(w)=1$.

For the sake of completeness we recall some properties of finite iterates of morphisms.

Lemma 3.4 [5]. Let $v \in M_{F}^{+} \backslash M^{*}, K=$ l.m. $(v)$ and $P=2(\sharp A) K$. The following statements are true:

(i) $h^{P+i}(v)=h^{P+n K+i}(v)$ for any $n \in \mathbb{N}$ and $i \in[0, K-1]$;

(ii) $h^{P+i}(v) \neq h^{P+n K+j}(v)$ for $i, j \in[0, K-1]$ and $i \neq j$.

The above properties are true for $S=\sharp A+(\sharp A) K$ in the place of $P$.

Lemma $3.5[5]$. For $a \in R^{r_{a}}, b \in L^{r_{b}}$ let us denote $P_{a}=\sharp A r_{a}, P_{b}=\sharp A r_{b}$. The following statements are true:

(i) $h^{P_{a}+i r_{a}}(a)$ is a prefix of $h^{P_{a}+(i+1) r_{a}}(a)$, for any $i \in \mathbb{N}$;

(ii) $h^{P_{b}+i r_{b}}(b)$ is a suffix of $h^{P_{b}+(i+1) r_{b}}(b)$, for any $i \in \mathbb{N}$.

The above properties are true for $S_{a}=\sharp M r_{a}, S_{b}=\sharp M r_{b}$ in the place of $P_{a}, P_{b}$, respectively.

\section{Some Results Characterizing $\delta L_{h}$}

If $a \in I_{R}^{r} \cup R^{r}$ then there exists the smallest integer $s_{a} \in[0, \sharp A]$ such that $h^{s_{a}}(a) \in w_{a} R^{r} A^{*}, w_{a} \in M_{F}^{*}$. In the following lemma the symbol $N_{a}$ is used to denote l.c.m.(r, l.m. $\left.\left(w_{a}\right)\right)$.

Lemma 4.1. Let $a \in I_{R}^{r} \cup R^{r}$. For an arbitrary (but fixed) $n \in \mathbb{N}$ we denote $w=h^{n+3(\sharp A) N_{a}}(a)$ and $(c, i)=$ first $_{E, \mathbb{Z}}(w)$. Let $j \in \mathbb{N}$ point out the (first) position of $c$ in $h^{N_{a}}(c)$.

The following statements are true:

(i) $\left(h^{N_{a}}\right)^{*, j}\left(w^{(i)}\right)$ is in $\delta L_{h}$ and so $\left[\left(h^{N_{a}}\right)^{*, j}\left(w^{(i)}\right)\right]$ is in $\delta L_{h} / \sigma^{*}$;

(ii) among all the possible words $w$ defined in the above manner for $n \in \mathbb{N}$ there exist exactly $N_{a}$ nonequivalent words and they are given by $n \in$ $\left[0, N_{a}-1\right]$. Hence there exist exactly $N_{a}$ mutually different equivalence classes in $\delta L_{h} / \sigma^{*}$ generated by these words.

Proof. According to the assumptions $h^{s_{a}}(a) \in w_{a} a_{1} A^{*}$ where $a_{1} \in R^{r}$. Hence denoting $t_{a}=\sharp A N_{a}+n-s_{a}$ we have $w \in h^{t_{a}+2 \sharp A N_{a}}\left(w_{a}\right) \cdot h^{t_{a}+2 \sharp A N_{a}}\left(a_{1}\right) A^{*}$. Now from Lemma 3.4 we obtain the equality

$$
h^{N_{a}}\left(h^{t_{a}+2 \sharp A N_{a}}\left(w_{a}\right)\right)=h^{t_{a}+2 \sharp A N_{a}}\left(w_{a}\right) .
$$


Lemma 3.5 implies that for any $p \in \mathbb{N}$ the word $h^{p N_{a}}\left(h^{t_{a}+2 \sharp A N_{a}}\left(a_{1}\right)\right)$ is a prefix of $h^{(p+1) N_{a}}\left(h^{t_{a}+2 \sharp A N_{a}}\left(a_{1}\right)\right)$. Thus $c$, the origin of $w$ and the first letter from $E$ which occurs in $w$, is also the first letter from $E$ in $h^{p N_{a}}\left(h^{t_{a}+2 \sharp A N_{a}}\left(a_{1}\right)\right)$ and also the first letter from $R^{r}$ in $w$. This implies that $w \in h^{t_{a}+2 \sharp A N_{a}}\left(w_{a}\right) h^{t_{a}+2 \sharp A N_{a}}\left(a_{1}\right) A^{*}$ is also in $L_{h}$.

Observe that for any $p \in \mathbb{N}$ the word $h^{p N_{a}}\left(h^{t_{a}+2 \sharp A N_{a}}\left(w_{a}\right) \cdot h^{t_{a}+2 \sharp A N_{a}}\left(a_{1}\right)\right)$ is a prefix of $h^{(p+1) N_{a}}\left(h^{t_{a}+2 \sharp A N_{a}}\left(w_{a}\right) \cdot h^{t_{a}+2 \sharp A N_{a}}\left(a_{1}\right)\right)$ and the word $h^{p N_{a}}(w) \in$ $h^{t_{a}+2 \sharp A N_{a}}\left(w_{a}\right) h^{p N_{a}}\left(h^{t_{a}+2 \sharp A N_{a}}\left(a_{1}\right)\right) A^{*}$ is also in $L_{h}$. This implies that there exists a limit word $\left(h^{N_{a}}\right)^{*, j}\left(w^{(i)}\right)$ which is right-infinite and it is in $\delta L_{h}$. Directly from the definition of $N_{a}$ it follows that it is the smallest positive integer such that $h^{N_{a}}\left(\left(h^{N_{a}}\right)^{*, j}\left(w^{(i)}\right)\right)=\left(h^{N_{a}}\right)^{*, j}\left(w^{(i)}\right)$. This means that among all the possible words $w$ defined in the above manner for $n \in \mathbb{N}$ there exist exactly $N_{a}$ nonequivalent words and they are given by $n \in\left[0, N_{a}-1\right]$.

Corollary 4.2. For $a, b \in I_{R}^{r} \cup R^{r}, n \in \mathbb{N}$ we denote $w_{n}^{a}=h^{n+3(\sharp A) N_{a}}(a)$ and $w_{n}^{b}=h^{n+3(\sharp A) N_{b}}(b)$. The counterpart of $w_{a} \in M_{F}^{*}$ for $b$ is denoted by $w_{b}$.

The following three conditions are equivalent:

(i) there exists $k \in\left[0, N_{b}-1\right]$ such that $h^{n+3(\sharp A) N_{a}-s_{a}}\left(w_{a}\right)=h^{n+3(\sharp A) N_{b}-s_{b}+k}$ $\left(w_{b}\right)$ and $\operatorname{first}_{E}\left(h^{n+3(\sharp A) N_{a}}(a)\right)=$ first $_{E}\left(h^{n+3(\sharp A) N_{b}+k}(b)\right)$;

(ii) the intersection of the sets $\left.\left\{\left(h^{N_{a}}\right)^{*,\left(i_{n}\right)}\left(w_{n}^{a}\right)\right)\right\}_{n \in\left[0, N_{a}-1\right]}$ and $\left\{\left(h^{N_{b}}\right)^{*,\left(j_{n}\right)}\right.$ $\left.\left.\left(w_{n}^{b}\right)\right)\right\}_{n \in\left[0, N_{b}-1\right]}$ is not empty;

(iii) the sets $\left.\left.\left\{\left[\left(h^{N_{a}}\right)^{*,\left(i_{n}\right)}\left(w_{n}^{a}\right)\right)\right]\right\}_{n \in\left[0, N_{a}-1\right]}, \quad\left\{\left[\left(h^{N_{b}}\right)^{*,\left(j_{n}\right)}\left(w_{n}^{b}\right)\right)\right]\right\}_{n \in\left[0, N_{b}-1\right]}$ coincide.

Proof. Assuming (i) observe that for a fixed $n$ there exists $k \in\left[0, N_{b}-1\right]$ such that $h^{n+3 \sharp A N_{a}-s_{a}}\left(w_{a}\right)=h^{n+3 \sharp A N_{b}-s_{b}+k}\left(w_{b}\right)$ and

$$
\operatorname{first}_{E}\left(h^{n+3 \sharp A N_{a}}(a)\right)=\operatorname{first}_{E}\left(h^{n+3 \sharp A N_{b}+k}(b)\right) \text {. }
$$

Analogously as in the proof of the above lemma we conclude that for any $q \in \mathbb{N}$

$$
\begin{aligned}
\operatorname{first}_{E}\left(h^{n+3 \sharp A N_{a}}(a)\right) & =\operatorname{first}_{E}\left(h^{q N_{a}}\left(h^{n-s_{a}+3 \sharp A N_{a}}\left(a_{1}\right)\right)\right) \\
& =\operatorname{first}_{E}\left(h^{n+3 \sharp A N_{b}+k}(b)\right) .
\end{aligned}
$$

Additionally

$$
h^{n+3 \sharp A N_{a}}(a), \quad h^{n+3 \sharp A N_{b}+k}(b) \in h^{t_{a}+2 \sharp A N_{a}}\left(w_{a}\right) \cdot h^{t_{a}+2 \sharp A N_{a}}\left(a_{1}\right) A^{*} .
$$

Thus

$$
\left.\left(h^{N_{b}}\right)^{*}\left(h^{n+3 \sharp A N_{b}+k}(b)\right) \in\left\{\left(h^{N_{a}}\right)^{*,\left(i_{n}\right)}\left(w_{n}^{a}\right)\right)\right\}_{n \in \mathbb{N}} .
$$

Finally according to the above lemma

$$
\left.\left(h^{N_{b}}\right)^{*}\left(h^{n+3 \sharp A N_{b}+k}(b)\right) \in\left\{\left(h^{N_{b}}\right)^{*,\left(j_{n}\right)}\left(w_{n}^{b}\right)\right)\right\}_{n \in \mathbb{N}} .
$$


The implication in the opposite direction is easy to prove. The equivalence of the conditions (ii) and (iii) is obvious.

Observation 4.3. The symmetric results for Lemma 4.1 and Corollary 4.2 are true for $a, b \in I_{L}^{r} \cup L^{r}$.

Notice that the symmetry mentioned above and in the sequel includes also the exchange of $\operatorname{first}_{E, \mathbb{Z}}(w)$ by last $E, \mathbb{Z}(w)$.

Example 4.4. From Example 3.2 follows that $a \in I_{R}^{2} \cup R^{2}$. We have $N_{a}=2$ and for $n=0,1$ there are two words $w=h^{0+3 \cdot 8 \cdot 2}(a)=a e^{48}$ and $\bar{w}=h^{1+3 \cdot 8 \cdot 2}(a)=b e^{49}$ for which $\operatorname{first}_{E, \mathbb{Z}}(w)=(a, 0)$ and $\operatorname{first}_{E, \mathbb{Z}}(\bar{w})=(b, 0)$ respectively and $j=0$ points out the first position of $a$ in $h^{2}(a)$ and the first position of $b$ in $h^{2}(b)$. Lemma 4.1 implies that $\left(h^{2}\right)^{*, 0}\left(w^{(0)}\right)=a e^{\omega}$ and $\left(h^{2}\right)^{*, 0}\left(\bar{w}^{(0)}\right)=b e^{\omega}$ are in $\delta L_{h}$. Observation 4.3 implies that also ${ }^{\omega} e a^{\prime}$ and ${ }^{\omega} e b^{\prime}$ are in $\delta L_{h}$.

If $a \in I_{R_{M}}^{r_{a}} \cup R_{M}^{r_{a}}$ then there exists the smallest integer $s_{a} \in[0, \sharp A]$ such that $h^{s_{a}}(a) \in w_{a} R_{M}^{r_{a}} A^{*}$, where $w_{a} \in M_{F}^{*}$. Hence for some $v_{a} \in M_{F}^{+} \backslash M^{*}$ we obtain $h^{s_{a}+r_{a}}(a) \in h^{r_{a}}\left(w_{a}\right) v_{a} R_{M}^{r_{a}} A^{*}$. In the lemma presented now the following notation is used: $M_{a}=$ l.m. $\left(w_{a} v_{a}\right), N_{a}=$ l.c.m. $\left(r_{a}, M_{a}\right), K_{a}=$ l.m. $\left(v_{a}\right)$.

Lemma 4.5. Let $a \in I_{R_{M}}^{r_{a}} \cup R_{M}^{r_{a}}$. For an arbitrary (but fixed) $n \in \mathbb{N}$ we denote $w=h^{n+3(\sharp A) N_{a}}(a)$ and $(c, i)=$ first $_{M_{R}, \mathbb{Z}}(w)$. Let $j \in \mathbb{N}$ point out the (first) position of $c$ in $h^{N_{a}}(c)$.

1. The following statements are true

(i) $\left(h^{N_{a}}\right)^{*, j}\left(w^{(i)}\right)$ is in $\delta L_{h}$;

(ii) among all the possible words $w$ defined in the above manner for $n \in$ $N$ there exist exactly $M_{a}$ nonequivalent words and they are given by $n \in\left[0, M_{a}-1\right]$. Hence there exist exactly $M_{a}$ mutually different equivalence classes in $\delta L_{h} / \sigma^{*}$ generated by these words.

2. Let $a \in I_{R_{M}}^{r_{a}} \cup R_{M}^{r_{a}}, b \in I_{R_{M}}^{r_{b}} \cup R_{M}^{r_{b}}$ and $w, v$ denote words obtained for $a, b$ respectively (in the manner as $w$ for a in the above). It is decidable, whether or not the words $\left(h^{N_{a}}\right)^{*, j}\left(w^{(i)}\right)$ and $\left(h^{N_{b}}\right)^{*, l}\left(v^{(k)}\right)$ are equivalent.

Proof. The fact that $a \in I_{R_{M}}^{r_{a}} \cup R_{M}^{r_{a}}$ implies that there exists the smallest integer $s_{a} \in[0, \sharp A]$ such that $h^{s_{a}}(a) \in w_{a} a_{1} A^{*}, a_{1} \in R_{M}^{r_{a}}, w_{a} \in M_{F}^{*}$. Hence $h^{s_{a}+r_{a}}(a) \in$ $h^{r_{a}}\left(w_{a}\right) v_{a} a_{1} A^{*}$, and $v_{a} \in M_{F}^{+} \backslash M^{*}$. By the definition of $R_{M}^{r_{a}}$ it follows that

$$
h^{s_{a}+r_{a}}(a) \in h^{r_{a}}\left(w_{a}\right) v_{a} a_{1} A^{*}=h^{r_{a}}\left(w_{a}\right) h^{r_{a}-1}\left(u_{0}\right) h^{r_{a}-2}\left(u_{1}\right) \ldots h^{0}\left(u_{r_{a}-1}\right) a_{1} A^{*}
$$

and if $i \in\left[0, r_{a}-1\right]$ then

$$
h^{i+1}\left(a_{1}\right) \in h^{i}\left(u_{0}\right) h^{i-1}\left(u_{1}\right) \cdot \ldots \cdot h^{0}\left(u_{i}\right) R_{M}^{r_{a}} A^{*},
$$

where $u_{i} \in M_{F}^{*}$.

Observe that because $v_{a} \in M_{F}^{+} \backslash M^{*}$ there exists $u_{i}$ such that $u_{i} \in M_{F}^{+} \backslash M^{*}$. Let $K_{a}=$ l.m. $\left(v_{a}\right)$. There exist numbers $k, l \in \mathbb{N}$ such that $N_{a}=k r_{a}=l K_{a}$. From 
the above we derive that

$$
\begin{aligned}
& h^{s_{a}+k r_{a}}(a) \in \\
& h^{N_{a}}\left(w_{a}\right) \cdot h^{(k-1) r_{a}+r_{a}-1}\left(u_{0}\right) \ldots h^{(k-1) r_{a}+0}\left(u_{r_{a}-1}\right) \cdot \ldots \cdot h^{r_{a}-1}\left(u_{0}\right) \ldots h^{0}\left(u_{r_{a}-1}\right) a_{1} A^{*}, \\
& h^{s_{a}+l K_{a}}(a) \in h^{N_{a}}\left(w_{a}\right) h^{(l-1) K_{a}+K_{a}-1}\left(u_{0}\right) \ldots h^{0}\left(u_{r_{a}-1}\right) \cdot a_{1} A^{*} .
\end{aligned}
$$

Now denoting

$$
y=h^{(k-1) r_{a}+r_{a}-1}\left(u_{0}\right) \ldots h^{(k-1) r_{a}+0}\left(u_{r_{a}-1}\right) \cdot \ldots \cdot h^{r_{a}-1}\left(u_{0}\right) \ldots h^{0}\left(u_{r_{a}-1}\right)
$$

we have for any $p \in \mathbb{N}$

$$
h^{s_{a}+p N_{a}}(a) \in h^{p N_{a}}\left(w_{a}\right) h^{(p-1) N_{a}}(y) h^{(p-2) N_{a}}(y) \ldots h^{0}(y) a_{1} A^{*} .
$$

Let us denote by $t_{a}=(\sharp A) N_{a}+n-s_{a}$ ( $n$ fixed). Thus for $p$ large enough we have

$$
\begin{aligned}
h^{n+p N_{a}}(a)= & h^{s_{a}+(p-(\sharp A)) N_{a}+\left(\sharp A N_{a}+n-s_{a}\right)}(a) \\
= & h^{s_{a}+(p-(\sharp A)) N_{a}+t_{a}}(a) \in h^{(p-\sharp A) N_{a}+t_{a}}\left(w_{a}\right) h^{(p-\sharp A-1) N_{a}+t_{a}}(y) . \\
& \cdot h^{(p-\sharp A-2) N_{a}+t_{a}}(y) \ldots h^{t_{a}}(y) \cdot h^{t_{a}-1}\left(u_{0}\right) \ldots h^{0}\left(u_{t}\right) R_{M}^{r_{a}} A^{*}
\end{aligned}
$$

for some $t \in\left[0, r_{a}-1\right]$.

From the assumptions it follows that

$$
h^{(3 \sharp A-\sharp A) N_{a}+t_{a}}\left(w_{a}\right) h^{(3 \sharp A-\sharp A-1) N_{a}+t_{a}}(y) \in\left(M_{R} \cup M\right)^{+} \backslash M^{*},
$$

and the word $\left(h^{(3 \sharp A-\sharp A) N_{a}+t_{a}}\left(w_{a}\right) h^{(3 \sharp A-\sharp A-1) N_{a}+t_{a}}(y)\right)$ is a prefix of $w$. Hence iterating $h^{N_{a}}$ on $w^{(i)}$ we obtain for $q=1,2 \ldots$

$$
\begin{aligned}
\left(h^{N_{a}}\right)^{q, j}\left(w^{(i)}\right) \in h^{(q+3 \sharp A-\sharp A) N_{a}+t_{a}}\left(w_{a}\right) h^{(q+3 \sharp A-\sharp A-1) N_{a}+t_{a}}(y) . \\
\cdot h^{(q+3 \sharp A-\sharp A-2) N_{a}+t_{a}}(y) \ldots h^{t_{a}}(y) h^{t_{a}-1}\left(u_{0}\right) \ldots h^{0}\left(u_{t}\right) R_{M}^{r_{a}} A^{*} .
\end{aligned}
$$

If $q \geq i$, then Lemma 3.4 implies that

$$
h^{(q+3 \sharp A-\# A-i) N_{a}+t_{a}}(y)=h^{2 \sharp A N_{a}+t_{a}}(y) \in u^{+}
$$

where $u \in A^{*}$ is a primitive word. Moreover it follows again from Lemma 3.4 that

$$
h^{(q+3 \sharp A-\sharp A) N_{a}+t_{a}}\left(w_{a}\right)=h^{2 \sharp A N_{a}+t_{a}}\left(w_{a}\right)
$$

and there exists a word $x \in A^{*}$ of minimal length such that $h^{2(\sharp A) N_{a}+t_{a}}\left(w_{a}\right) \in x u^{*}$. Hence there exist the limit word $\left(h^{N_{a}}\right)^{*, j}\left(w^{(i)}\right) \in x u \ldots u \ldots$ This word is rightinfinite and it is in $\delta L_{h}$ and so its equivalence class is in $\delta L_{h} / \sigma^{*}$. 
Observe that (Lem. 3.4) $h^{M_{a}}\left(h^{2(\sharp A) N_{a}+t_{a}}(y)=h^{2(\sharp A) N_{a}+t_{a}}(y)\right.$ which implies that $h^{M_{a}}(u)=u$ where $M_{a}$ is the smallest integer such that $h^{M_{a}}(x u)=x u$. Taking into account the fact that no letters from $E \cup I_{E}$ occur in $x u$ we conclude that $M_{a}$ is the smallest integer such that $h^{M_{a}, j}\left(\left(h^{N_{a}}\right)^{*, j}\left(w^{(i)}\right)\right)=\left(h^{N_{a}}\right)^{*, j}\left(w^{(i)}\right)$ which implies finally that among all the possible words $w$ defined in the above manner for $n \in \mathbb{N}$ there exist exactly $M_{a}$ nonequivalent words and they are given by $n \in\left[0, M_{a}-1\right]$.

Now let us consider two words:

$$
\left(h^{N_{a}}\right)^{*, j}\left(w^{(i)}\right)=\left(h^{N_{a}}\right)^{*, j}\left(h^{n_{a}+3(\sharp A) N_{a}}(a)^{(i)}\right)=x_{a} u_{a}^{\omega}
$$

and

$$
\left(h^{N_{b}}\right)^{*, l}\left(v^{(k)}\right)=\left(h^{N_{b}}\right)^{*, l}\left(h^{n_{b}+3(\sharp A) N_{b}}(b)^{(k)}\right)=x_{b} u_{b}^{\omega} .
$$

There are two possible cases:

(1) if $u_{a}$ and $u_{b}$ are not conjugate words then $\left(h^{N_{a}}\right)^{*, j}\left(w^{(i)}\right)$ and $\left(h^{N_{b}}\right)^{*, l}\left(v^{(k)}\right)$ are not equivalent also;

(2) if $u_{a} \approx u_{b}$ then there exists a word $u_{R}$ such that $u_{R} u_{a}$ is a prefix of $u_{b} u_{b}$. There exists a word $y_{b}$ of minimal length and such that $x_{b} u_{R} \in y_{b} u_{a}^{*}$. Thus $\left.\left.\left(h^{N_{a}}\right)^{*, j}(w)^{(i)}\right)=\left(h^{N_{b}}\right)^{*, l}(v)^{(k)}\right)$ if and only if $x_{a}=y_{b}$.

Hence in the both cases the equivalency of the considered words is decidable.

Corollary 4.6. For $a \in I_{R_{M}}^{r_{a}} \cup R_{M}^{r_{a}}, b \in I_{R_{M}}^{r_{b}} \cup R_{M}^{r_{b}}$ and $n, m \in \mathbb{N}$ we denote $w_{n}=h^{n+3(\sharp A) N_{a}}(a)$ and $v_{m}=h^{m+3(\sharp A) N_{b}}(b)$. The following two conditions are equivalent:

(i) the intersection of the sets $\left\{\left(h^{N_{a}}\right)^{*, j}\left(w_{n}\right)\right\}_{n \in\left[0, M_{a}-1\right]}$, and $\left\{\left(h^{N_{b}}\right)^{*, l}\right.$ $\left.\left(v_{m}\right)\right\}_{m \in\left[0, M_{b}-1\right]}$ is not empty;

(ii) the sets $\left\{\left[\left(h^{N_{a}}\right)^{*, j}\left(w_{n}\right)\right]\right\}_{n \in\left[0, M_{a}-1\right]}$ and $\left\{\left[\left(h^{N_{b}}\right)^{*, j}\left(v_{m}\right)\right]\right\}_{m \in\left[0, M_{b}-1\right]}$ coincide and $M_{a}=M_{b}$.

Proof. Assuming (i) we have equality $h^{N_{a}, j}\left(w_{n}\right)=h^{N_{b}, l}\left(v_{m}\right)=x_{a} u_{a}^{\omega}$. Arguing in the similar way as in the above lemma we find the smallest integers $M_{a}, M_{b}$ such that $h^{M_{a}}\left(x_{a} u_{a}\right)=x_{a} u_{a}$ and $h^{M_{b}}\left(x_{a} u_{a}\right)=x_{a} u_{a}$. Lemma 3.3 implies that $M_{a}=M_{b}$. The result 1 in the above lemma finishes the proof of the implication $(i) \Rightarrow(i i)$. The implication in the opposite direction is obvious.

Observation 4.7. The symmetric results for Lemma 4.5 and Corollary 4.6 are true for $a \in I_{L_{M}}^{r_{a}} \cup L_{M}^{r_{a}}$.

Example 4.8. From Example 3.2 follows that $a^{\prime} \in I_{R_{M}}^{2} \cup R_{M}^{2}$. We have $N_{a^{\prime}}=2$, $M_{a^{\prime}}=1$ and for $n=0$ there is a word $w=h^{0+3 \cdot 8 \cdot 2}\left(a^{\prime}\right)=e^{48} a^{\prime}$ for which first $_{M_{R}, \mathbb{Z}}(w)=(e, 0)$ and $j=0$ points out the first position of $e$ in $h^{2}(e)$. Lemma 4.5 implies that $\left(h^{2}\right)^{*, 0}\left(w^{(0)}\right)=e e^{\omega}$ is in $\delta L_{h}$. Observation 4.7 implies that also ${ }^{\omega} e e$ is in $\delta L_{h}$. 
From Lemmas 3.1 and 3.3 it follows that the set $L_{M} R_{M}=\left\{a u b \in A^{*}: \exists c \in\right.$ $\left.E \cup I_{E}, h(c) \in A^{*} a u b A^{*}, a \in I_{L_{M}}^{r_{a}} \cup L_{M}^{r_{a}}, b \in I_{R_{M}}^{r_{b}} \cup R_{M}^{r_{b}}, u \in M_{F}^{*}\right\}$ is finite and effectively constructable.

Now let us consider $a u b \in L_{M} R_{M}$, where $a, b \in A$. One can find the smallest integers $s_{a}, s_{b} \in[0, \sharp A]$ such that $h^{s_{a}}(a) \in A^{*} L_{M}^{r_{a}} w_{a}$ and $h^{s_{b}}(b) \in w_{b} R_{M}^{r_{b}} A^{*}$ where $w_{a}, w_{b} \in M_{F}^{*}$. Hence $h^{s_{a}+r_{a}}(a) \in A^{*} L_{M}^{r_{a}} v_{a} h^{r_{a}}\left(w_{a}\right)$ and $h^{s_{b}+r_{b}}(b) \in$ $h^{r_{b}}\left(w_{b}\right) v_{b} R_{M}^{r_{b}} A^{*}$, where $v_{a}, v_{b} \in M_{F}^{+} \backslash M^{*}$.

We use in the sequel the following notations.

$$
\begin{array}{r}
K_{a}=\text { l.m. }\left(v_{a}\right), K_{b}=\text { l.m. }\left(v_{b}\right), \quad M_{a b}=\text { l.c.m. }\left(K_{a}, \operatorname{l.m} \cdot\left(w_{a} u w_{b}\right), K_{b}\right), \\
\text { and } N_{a b}=\text { l.c.m. }\left(r_{a}, r_{b}, M_{a b}\right) .
\end{array}
$$

Thus for any $n \in\left[0, N_{a b}-1\right]$ we have $h^{n+3(\sharp A) N_{a b}}(a u b) \in A^{*} L_{M}^{r_{a}} m R_{M}^{r_{b}} A^{*}$, where $m \in M_{F}^{+} \backslash M^{*}$.

Lemma 4.9. Let $a u b \in L_{M} R_{M}$. For an arbitrary (but fixed) $n \in \mathbb{N}$ we denote $w=h^{n+3(\sharp A) N_{a b}}(a u b)$ and $(c, i)=$ first $_{M_{R}, \mathbb{Z}}\left(h^{n+3(\sharp A) N_{a b}}(b)\right)$. Let $k \in \mathbb{N}$ point out the (first) position of the letter $c$ in $w$, that is, $k=\left|h^{n+3(\sharp A) N_{a b}}(a u)\right|+i$. Finally let $j \in \mathbb{N}$ point out the first position of the letter $c$ in $h^{N_{a b}}(c)$.

1. The following statements are true:

(i) $\left(h^{N_{a b}}\right)^{*, j}\left(w^{(k)}\right)$ is in $\delta L_{h}$;

(ii) it is decidable, whether or not a word obtained in (i) is periodic;

(iii) if for some $n \in\left[0, M_{a b}-1\right]$ the word $\left(h^{N_{a b}}\right)^{*, j}\left(w^{(k)}\right)$ is not periodic then among all the possible words $\left(h^{N_{a b}}\right)^{*, j}\left(w^{(k)}\right)$ defined in the above manner for $n \in N$ there exist exactly $M_{a b}$ nonequivalent words and they are given by $n \in\left[0, M_{a b}-1\right]$. Hence there exist exactly $M_{a b}$ mutually different equivalence classes in $\delta L_{h} / \sigma^{*}$ generated by these words.

2. For aub, dve $\in L_{M} R_{M}$ denote $w=h^{n+3(\sharp A) N_{a b}}(a u b)$ and $v=h^{m+3(\sharp A) N_{d e}}($ dve $)$. It is decidable, whether or not two words $\left(h^{N_{a b}}\right)^{*, j}\left(w^{(k)}\right)$ and $\left(h^{N_{d e}}\right)^{*, p}\left(v^{(r)}\right)$ are equivalent. Hence it is decidable, whether or not two equivalence classes $\left[\left(h^{N_{a b}}\right)^{*, j}\left(w^{(k)}\right)\right]$ and $\left[\left(h^{N_{d e}}\right)^{*, p}\left(v^{(r)}\right)\right]$ coincide.

Proof. We apply to the letters $a, b$ the argumentation from Lemma 4.5 and come to the following conclusions.

If $p$ is large enough then denoting

$$
y=h^{\left(i_{a b}-1\right) r_{b}+r_{b}-1}\left(v_{0}\right) \ldots h^{\left(i_{a b}-1\right) r_{b}+0}\left(v_{r_{b}-1}\right) \cdot \ldots \cdot h^{r_{b}-1}\left(v_{0}\right) \ldots h^{0}\left(v_{r_{b}-1}\right)
$$

we have for some $t \in\left[0, r_{b}-1\right]$.

$$
\begin{aligned}
h^{n+p N_{a b}}(b)= & h^{s_{b}+(k-\# A) N_{a b}+\left(\# A N_{a b}+n-s_{b}\right)}(b) \\
= & h^{s_{b}+(k-\# A) N_{a b}+t_{b}}(b) \in h^{(k-Q) N_{1}+t_{b}}\left(w_{b}\right) h^{(k-Q-1) N_{1}+t_{b}}(y) . \\
& \cdot h^{(k-Q-2) N_{1}+t_{b}}(y) \ldots h^{0+t_{b}}(y) \cdot \ldots \cdot h^{t_{b}-1}\left(v_{0}\right) \ldots h^{0}\left(v_{t}\right) R_{M}^{r_{b}} A^{*} .
\end{aligned}
$$


If $q$ is large enough then denoting

$$
z=h^{0}\left(u_{r_{a}-1}\right) \ldots h^{r_{a}-1}\left(u_{0}\right) \cdot \ldots \cdot h^{\left(j_{a b}-1\right) r_{a}+0}\left(u_{r_{a}-1}\right) \ldots h^{\left(j_{a b}-1\right) r_{a}+r_{a}-1}\left(u_{0}\right)
$$

we have for some $s \in\left[0, r_{b}-1\right]$

$$
\begin{aligned}
h^{n+q N_{a b}}(a) \in A^{*} L_{M}^{r_{a}} h^{0}\left(u_{s}\right) \ldots h^{0+t_{a}-1}\left(u_{0}\right) h^{t_{a}}(z) \cdot \ldots \cdot h^{(k-\sharp A-2) N_{a b}+t_{a}}(z) . \\
\cdot h^{(k-\sharp A-1) N_{a b}+t_{a}}(z) h^{(k-\sharp A) N_{a b}+t_{a}}\left(w_{a}\right) .
\end{aligned}
$$

Applying again the argumentation from Lemma 4.5 we obtain two primitive words $u_{b}, u_{a}$ and words $x_{b}, x_{a}$ of the minimal length such that $h^{2 \sharp A N_{1}+t_{b}}\left(w_{b}\right) \in x_{b} u_{b}^{*}$ and $h^{2 \sharp A N_{1}+t_{a}}\left(w_{a}\right) \in u_{a}^{*} x_{a}$. The equality $h^{n+(j+3 \sharp A) N_{1}}(u)=h^{n+3 \sharp A N_{1}}(u)$ (Lem. 3.4) finally leads to the conclusion that there exist the limit word $\left(h^{N_{a b}}\right)^{*, j}\left(w^{(k)}\right)$. There exists $f \in E \cup I_{E}$ such that $h(f)=u_{L} a u b u_{R}$ where $u_{L}, u_{R} \in A^{*}$. This means that there exists the limit word

$$
\lim _{\alpha \rightarrow \infty}\left(h^{N_{a b}}\right)^{\alpha, j}\left(h^{n+3(\sharp A) N_{a b}}\left(u_{L}\right)\left(w^{(k)}\right) h^{n+3(\sharp A) N_{a b}}\left(u_{R}\right)\right) \in{ }^{\omega} u_{a} x_{a} y_{u} x_{b} u_{b} u_{b}^{\omega}
$$

and these two limits are equal. Hence $\left(h^{N_{a b}}\right)^{*, j}\left(w^{(k)}\right)$ is bi-infinite and it is in $\partial L_{h}$. It can be factorized in the form $\left(h^{N_{a b}}\right)^{*, j}\left(w^{(k)}\right)=v_{L} h^{n+3(\sharp A) N_{a b}}(u) x_{b} u_{b} u_{b}^{\omega}$. The words $v_{L}$ and $x_{b} u_{b} u_{b}^{\omega}$ are left and right-infinite and according to the previous lemma are in $\delta L_{h}$ and so is $\left(h^{N_{a b}}\right)^{*, j}\left(w^{(k)}\right)$.

The fact that among all the possible words $w$ defined in the above manner for $n \in \mathbb{N}$ there exist exactly $M_{a b}$ nonequivalent words and they are given by $n \in\left[0, M_{a}-1\right]$ can be proved in a similar way as in Lemma 4.5.

For the word $\left(h^{N_{a b}}\right)^{*, j}\left(w^{(k)}\right)$ let us consider two possible cases:

(1) if $u_{a} \not \approx u_{b}$ (not conjugates) then $\left(h^{N_{a b}}\right)^{*, j}\left(w^{(k)}\right)$ is not a periodic word;

(2) if $u_{a} \approx u_{b}$ then there exists a word $z_{L}$ such that $u_{b} z_{L}$ is a suffix of $u_{a} u_{a}$. Thus $\left(h^{N_{a b}}\right)^{*, j}\left(w^{(k)}\right)$ is periodic if and only if $z_{L} x_{a} y_{u} x_{b} \in u_{b}^{*}$.

From the above considerations and Lemma 3.4 we conclude that it is decidable whether or not the word $\left(h^{N_{a b}}\right)^{*, j}\left(w^{(k)}\right)$ is periodic, which proves (ii). If $\left(h^{N_{a b}}\right)^{*, j}\left(w^{(k)}\right)$ is not a periodic word then from (ii) and Lemma 3.4 there exist no numbers $n_{1}, n_{2} \in\left[0, M_{a b}-1\right], n_{1} \neq n_{2}$ such that $\left(h^{N_{a b}}\right)^{*, j}\left(h^{n_{1}+3(\sharp A) N_{a b}}\right)^{(k)}(a u b) \in$ $\left[\left(h^{N_{a b}}\right)^{*, j}\left(h^{n_{2}+3(\sharp A) N_{a b}}\right)^{(k)}(a u b)\right]$ which proves (iii).

It remains to prove 2 . It is decidable whether or not two equivalence classes of words: $\left(h^{N_{a b}}\right)^{*, j}\left(w^{(k)}\right) \in{ }^{\omega} u_{a} x_{a} y_{u} x_{b} u_{b} u_{b}^{\omega}$ and $\left(h^{N_{d e}}\right)^{*, p}\left(v^{(r)}\right) \in{ }^{\omega} u_{c} x_{c} y_{v} x_{d} u_{d} u_{d}^{\omega}$ are equal. Namely:

(1) if $u_{a} \not \approx u_{c}$ or $u_{b} \not \approx u_{d}$ then the equality does not hold;

(2) if $u_{a}$ and $u_{c}$ are equivalent or $u_{b}$ and $u_{d}$ are equivalent and the words $\left(h^{N_{a b}}\right)^{*, j}\left(w^{(k)}\right)$ and $\left(h^{N_{d e}}\right)^{*, p}\left(v^{(r)}\right)$ are periodic, then the two equivalence classes are equal;

(3) if $u_{a}, u_{c}$ are equivalent and $u_{b}, u_{d}$ are equivalent then there exist words $u_{L}, u_{R}$ such that $u_{a} u_{L}$ is a suffix of $u_{c} u_{c}$ and $u_{R} u_{b}$ is a prefix of $u_{d} u_{d}$. Hence 
$\left(h^{N_{d e}}\right)^{*, p}\left(v^{(r)}\right) \in{ }^{\omega} u_{a} u_{L} x_{c} y_{v} x_{d} u_{R} u_{b}^{\omega}$ and there exist words $x_{R}, y_{R}$ of the minimal length such that $x_{a} y_{u} x_{b} \in x_{R} u_{b}^{*}$ and $u_{L} x_{c} y_{v} x_{d} u_{R} \in y_{R} u_{b}^{*}$. There exist also $x_{L}, y_{L}$ of the minimal length such that $x_{R} \in u_{a}^{*} x_{L}$ and $y_{R} \in$ $u_{a}^{*} y_{L}$. Now taking into account that in the considered case at least one of the words $\left(h^{N_{a b}}\right)^{*, j}\left(w^{(k)}\right),\left(h^{N_{d e}}\right)^{*, p}\left(v^{(r)}\right)$ is not periodic we conclude that the equivalence classes are equal if and only if $x_{L}=y_{L}$. This completes the proof of the lemma.

Example 4.10. We have $L_{M} R_{M}=\left\{a e a^{\prime}\right\}, M_{a a^{\prime}}=1, N_{a a^{\prime}}=2$. For $n=0$ there is a word $w=h^{0+3 \cdot 8 \cdot 2}\left(a e a^{\prime}\right)=a e^{48} e e^{48} a^{\prime}$ for which first $M_{R}, \mathbb{Z}\left(h^{48}\left(a^{\prime}\right)\right)=(e, 0)$, $k=50$ and $j=0$ points out the first position of $e$ in $h^{2}(e)$. Lemma 4.9 implies that $\left(h^{2}\right)^{*, 0}\left(w^{(50)}\right)={ }^{\omega} e e e^{\omega}$ is in $\delta L_{h}$.

Let us introduce two sets

$$
\begin{gathered}
L_{M} R=\left\{a u b \in A^{*}: \exists c \in E \cup I_{E}, h(c) \in A^{*} a u b A^{*},\right. \\
\left.a \in I_{L_{M}}^{r_{a}} \cup L_{M}^{r_{a}}, b \in I_{R}^{r_{b}} \cup R^{r_{b}}, u \in M_{F}^{*}\right\}, \\
L R_{M}=\left\{a u b \in A^{*}: \exists c \in E \cup I_{E}, h(c) \in A^{*} a u b A^{*},\right. \\
\left.a \in I_{L}^{r_{a}} \cup L^{r_{a}}, b \in I_{R_{M}}^{r_{b}} \cup R_{M}^{r_{b}}, u \in M_{F}^{*}\right\} .
\end{gathered}
$$

From Lemmas 3.1 and 3.3 these sets are finite and effectively constructable.

Now let $a u b \in L_{M} R, a, b \in A$. There exists $f \in E \cup I_{E}$, such that $h(f) \in$ $A^{*} a u b A^{*}, u \in M_{F}^{*}$. One can find the smallest integers $s_{a}, s_{b} \in[0, \sharp A]$ such that $h^{s_{a}}(a) \in A^{*} L_{M}^{r_{a}} w_{a}, h^{s_{b}}(b) \in w_{b} R^{r_{b}} A^{*}$, where $w_{a}, w_{b} \in M_{F}^{*}$. Moreover $h^{s_{a}+r_{a}}(a) \in$ $A^{*} L_{M}^{r_{a}} v_{a} h^{r_{a}}\left(w_{a}\right)$ where $v_{a} \in M_{F}^{+} \backslash M^{*}$.

Let $M_{a b}=$ l.c.m.(l.m. $\left.\left(v_{a} w_{a} u w_{b}\right), r_{b}\right), N_{a b}=$ l.c.m. $\left(r_{a}, M_{a b}\right)$. Hence for any $n \in\left[0, N_{a b}-1\right]$ it holds $h^{n+3(\sharp A) N_{a b}}(a u b) \in A^{*} L_{M}^{r_{a}} m R^{r_{b}} A^{*}$, where $m \in M_{F}^{+} \backslash M^{*}$.

Lemma 4.11. Let aub $\in L_{M} R$. For arbitrary (but fixed) $n \in \mathbb{N}$ we denote $w=$ $h^{n+3(\sharp A) N_{a b}}(a u b)$ and $(c, i)=$ first $_{E, \mathbb{Z}}\left(h^{n+3(\sharp A) N_{a b}}(b)\right)$. Let $k \in \mathbb{N}$ point out the position of the letter $c$ in $w$, that is, $k=\left|h^{n+3(\sharp A) N_{a b}}(a u)\right|+i$. Finally let $j \in \mathbb{N}$ point out the first position of the letter $c$ in $h^{N_{a b}}(c)$.

1. The following statements are true

(i) $\left(h^{N_{a b}}\right)^{*, j}\left(w^{(k)}\right)$ is in $\delta L_{h}$;

(ii) among all the possible words $w$ defined in the above manner for $n \in N$ there exist exactly $M_{a b}$ nonequivalent words and they are given by $n \in$ $\left[0, M_{a b}-1\right]$. Hence there exist exactly $M_{a b}$ mutually different equivalence classes in $\delta L_{h} / \sigma^{*}$ generated by these words.

2. For aub, dve $\in L_{M} R$ denote $w=h^{n+3(\sharp A) N_{a b}}(a u b)$ and $v=h^{m+3(\sharp A) N_{d e}}($ dve). It is decidable, whether or not two words $\left(h^{N_{a b}}\right)^{*, j}\left(w^{(k)}\right)$ and $\left(h^{N_{d e}}\right)^{*, p}\left(v^{(r)}\right)$ are equivalent. Hence it is decidable, whether or not two equivalence classes $\left[\left(h^{N_{a b}}\right)^{*, j}\left(w^{(k)}\right)\right]$ and $\left[\left(h^{N_{d e}}\right)^{*, p}\left(v^{(r)}\right)\right]$ coincide.

Proof. Denote by $t_{a}=(\sharp A) N_{a b}+n-s_{a}, t_{b}=(\sharp A) N_{a b}+n-s_{b}$ for a fixed $n$. By the definition of $N_{a b}$ there exists a number $j_{a b} \in \mathbb{N}$ such that $N_{a b}=j_{a b} r_{a}$. It is clear 
that $h^{s_{a}}(a) \in A^{*} a_{1} w_{a}, a_{1} \in L_{M}^{r_{a}}, h^{s_{b}}(b) \in w_{b} b_{1} A^{*}, b_{1} \in R^{r_{b}}$ and if $i \in\left[0, r_{a}-1\right]$ then $h^{i+1}\left(a_{1}\right) \in A^{*} L_{M}^{r_{a}} h^{0}\left(u_{i}\right) h^{1}\left(u_{i-1}\right) \cdot \ldots \cdot h^{i-1}\left(u_{1}\right) h^{i}\left(u_{0}\right)$, where $u_{i} \in M_{F}^{*}$.

The fact that $v_{a} \in M_{F}^{+} \backslash M^{*}$ implies that there exists $j \in\left[0, r_{a}-1\right]$ such that $u_{j} \in M_{F}^{+} \backslash M^{*}$.

Now let $y=h^{0}\left(u_{r_{a}-1}\right) \cdot \ldots \cdot h^{\left(j_{a b}-1\right) r_{a}+r_{a}-1}\left(u_{0}\right)$, where $u_{i} \in M_{F}^{*}$. Thus for $p$ large enough

$$
\begin{aligned}
h^{n+p N_{a b}}(a) \in A^{*} L_{M}^{r_{a}} h^{0}\left(u_{l}\right) \ldots h^{0+t_{a}-1}\left(u_{0}\right) h^{t_{a}}(y) \cdot \ldots \cdot h^{(p-(\sharp A)-2) N_{a b}+t_{a}}(y) . \\
\cdot h^{(p-(\sharp A)-1) N_{a b}+t_{a}}(y) h^{(p-(\sharp A)) N_{a b}+t_{a}}\left(w_{a}\right) .
\end{aligned}
$$

Hence for $g \in \mathbb{N}$

$$
\begin{aligned}
& \left(h^{\left.N_{a b}\right)^{g, j}}\left(w^{(k)}\right) \in A^{*} L_{M}^{r_{a}} h^{0}\left(u_{l}\right) \ldots h^{0+t_{a}-1}\left(u_{0}\right) .\right. \\
& \cdot h^{t_{a}}(y) \cdot \ldots \cdot h^{(g+3(\# A)-(\sharp A)-2) N_{a b}+t_{a}}(y) h^{(g+3(\# A)-(\sharp A)-1) N_{a b}+t_{a}}(y) . \\
& \cdot h^{(g+3(\# A)-(\sharp A)) N_{a b}+t_{a}}\left(w_{a}\right) \ldots h^{n+\left(g+3(\# A) N_{a b}\right.}(u) h^{(g+2(\# A)) N_{a b}+t_{b}}\left(w_{b}\right) . \\
& \cdot\left(h^{n+3(\sharp A) N_{a b}}(b)\right)^{(i)} A^{*} .
\end{aligned}
$$

Note that according to Lemma 3.4 it holds for $g \geq i$

$$
h^{(g+3(\sharp A)-(\sharp A)-i) N_{a b}+t_{a}}(y)=h^{2(\sharp A) N_{a b}+t_{a}}(y) \in u^{+}
$$

where $u$ is a primitive word.

Denoting

$$
z=h^{t_{a}+2(\sharp A) N_{a b}}\left(w_{a}\right) h^{n+3(\sharp A) N_{a b}}(u) h^{t_{b}+2(\sharp A) N_{a b}}\left(w_{b}\right) .
$$

Lemma 3.4 implies that

$$
h^{(g+3(\sharp A)-(\sharp A)) N_{a b}+t_{a}}\left(w_{a}\right) h^{n+(g+3(\sharp A)) N_{a b}}(u) h^{(g+2(\sharp A)) N_{a b}+t_{b}}\left(w_{b}\right)=z .
$$

Hence the sequence $\left(h^{N_{a b}}\right)^{g, j}\left(w^{(k)}\right)$ converges to $\left(h^{N_{a b}}\right)^{*, j}\left(w^{(k)}\right)$ when $g \rightarrow \infty$.

As mentioned in the above there exists $f \in E \cup I_{E}$, such that $h(f) \in u_{L} a u b u_{R}$, $u \in M_{F}^{*}, u_{L}, u_{R} \in A^{*}$. This implies that from the sequence of equivalence classes $\left\{\left(h^{N_{a b}}\right)^{g}\left(h^{n+N_{a b}}\left(u_{L}\right) h^{n+N_{a b}}(a u b) h^{n+N_{a b}}\left(u_{R}\right)\right)\right\}_{g \in \mathbb{N}}$ one can choose the sequence of words with origins $c$ and all the words from the sequence are in $L_{h}$. We have

$$
\lim _{g \rightarrow \infty}\left(h^{N_{a b}}\right)^{g, j}\left(w^{(k)}\right)={ }^{\omega} u z\left(h^{N_{a b}}\right)^{*, j}\left(h^{t_{b}+2(\sharp A) N_{a b}}\left(b_{1}\right)^{(i)}\right) .
$$

The limit word is bi-infinite and is in $\partial L_{h}$. Now observe that

$$
{ }^{\omega} u z\left(h^{N_{a b}}\right)^{*, j}\left(h^{t_{b}+2(\sharp A) N_{a b}}\left(b_{1}\right)^{(i)}\right)=v_{L} m_{C} v_{R}
$$


where

$$
m_{C}=h^{n+3(\sharp A) N_{a b}}(u) \in\left(M \cup M_{R}\right)^{*}
$$

and

$$
v_{L} \quad \text { and } \quad v_{R}=h^{t_{b}+2(\sharp A) N_{a b}}\left(w_{b}\right)\left(h^{N_{a b}}\right)^{*, j}\left(h^{t_{b}+2(\sharp A) N_{a b}}\left(b_{1}\right)^{(i)}\right)
$$

are left-infinite and right-infinite words. According to Lemmas 4.1 and 4.5 these words are in $\delta L_{h}$. It implies finally that $\left(h^{N_{a b}}\right)^{*, j}\left(w^{(k)}\right)$ is a pasted word, an element of $\delta L_{h}$.

Taking into account that for every $n \in \mathbb{N}$ any of the words in (i) is of the form $\omega^{\omega} u z\left(h^{N_{a b}}\right)^{*, j}\left(h^{t_{b}+2(\sharp A) N_{a b}}\left(b_{1}\right)^{(i)}\right)$ and that $a l p h_{E \cup I_{E}}(u z)=\emptyset$ the justification of (ii) follows.

For the proof of the statement 2 consider two words: $w=h^{n+3(\sharp A) N_{a b}}(a u b)$ and $v=h^{m+3(\sharp A) N_{d e}}(d v e)$. As above there exist $u_{a}, z_{a}, v_{d}, z_{d}$ such that $w=$ $\omega u_{a} z_{a}\left(h^{N_{a b}}\right)^{*, j}\left(h^{t_{b}+2(\sharp A) N_{a b}}\left(b_{1}\right)^{(i)}\right)$ and $v={ }^{\omega} u_{d} z_{d}\left(h^{N_{d e}}\right)^{*, p}\left(h^{t_{e}+2(\sharp A) N_{d e}}\left(e_{1}\right)^{(r)}\right)$.

If $w$ and $v$ are equal then $u_{a}$ and $u_{d}$ have to be equal. If the last equality is true then there exists a word $u_{L}$ such that $u_{a} u_{L}$ is a suffix of $u_{d} u_{d}$. There exists also a word $y_{R}$ such that the length of $y_{R}$ is minimal and $u_{L} z_{d} \in u_{a}^{*} y_{R}$. From Lemmas 3.3, 3.4 and properties of primitive words it follows that $w=v$ if and only if $z_{R}=y_{R}$ and first $\left(h^{t_{b}+2(\sharp A) N_{a b}}\left(b_{1}\right)\right)=$ first $\left(h^{t_{e}+2(\sharp A) N_{d e}}\left(e_{1}\right)\right.$. Hence the decidability stated in 2 is proved.

Observation 4.12. The symmetric results for Lemma 4.11 hold for $a u b \in L R_{M}$.

Example 4.13. We have $L_{M} R=\{a e a\}, M_{a a}=2, N_{a a}=2$. For $n=0,1$ there are two words $w=h^{0+3 \cdot 8 \cdot 2}($ aea $)=a e^{48} e a e^{48}$ and $\bar{w}=h^{1+3 \cdot 8 \cdot 2}(a)=b e^{49} e b e^{49}$ for which first $_{E, \mathbb{Z}}\left(h^{48}(a)\right)=(a, 0), k=50$ and $j=0$ points out the first position of $a$ in $h^{2}(a)$ and $b$ in $h^{2}(b)$ respectively. Lemma 4.11 implies that $\left(h^{2}\right)^{*, 0}\left(w^{(50)}\right)={ }^{\omega} e a e^{\omega}$ and $\left(h^{2}\right)^{*, 0}\left(\bar{w}^{(50)}\right)={ }^{\omega} e b e^{\omega}$ are in $\delta L_{h}$.

The following two lemmas are from the paper Matyja [7]

Lemma 4.14 [7]. Let $a \in R^{r}$ and $w \in A^{*}$ be a primitive word. Let $j \in \mathbb{N}$ denote the first position of a in $h^{r}(a)$.

$\left(h^{r}\right)^{*, j}\left(a^{(0)}\right)=w^{(j)} w^{\omega}$ if and only if

(i) $h^{2(\sharp A) r}(a)=u_{0} a u_{1} a z$, where a does not occur in $u_{0} u_{1}$;

(ii) $h^{(\sharp A-1) r}\left(a u_{1}\right) \in w^{+}$;

(iii) $h^{r}(w) \in w^{+} w$.

Now let us denote by $P^{r}$ a subset of primitive words defined as follows.

$$
P^{r}=\left\{w \in A^{+} \mid w \text { primitive, } \operatorname{first}_{R^{r}, \mathbb{Z}}(w)=(a, t),\left(h^{r}\right)^{*, t}\left(a^{(0)}\right)=w^{(t)} w^{\omega}\right\}
$$

The set $P=\bigcup_{r \in[1, \sharp A]} P^{r}$ is finite and effectively constructable what can be derived from Lemmas 3.1, 3.2 and the previous lemma. 
Lemma 4.15 [7]. Let $v \in P^{r}$ and $h: A^{*} \rightarrow A^{*}$ be a morphism.

(i) $r$ is the smallest integer, such that $h^{r}(v) \in v^{+} v$.

(ii) Let $a \in R^{r_{a}} \cap \operatorname{alph}_{E}(v)$ and $t \in \mathbb{N}$ point out the position of the letter $a$ in the word $h^{r_{a}}\left(a^{(0)}\right)$. Then the words $\left(h^{r_{a}}\right)^{*, t}\left(a^{(0)}\right)$ and $y^{(0)} x(y x)^{\omega}$ are equal modulo the origin, where $v=x y$.

Observation 4.16. The symmetric results for Lemma 4.15 holds for $a \in L^{r_{a}}$.

From Lemmas 3.1 and 3.2 the set $L R=\left\{a u b \in A^{*}: \exists c \in E \cup I_{E}, h(c) \in\right.$ $\left.A^{*} a u b A^{*}, a \in I_{L}^{r_{a}} \cup L^{r_{a}}, b \in I_{R}^{r_{b}} \cup R^{r_{b}}, u \in M_{F}^{*}\right\}$ is finite end effectively constructable.

For $a u b \in L R$ there exist the smallest integers $s_{a}, s_{b} \in[0, \sharp A]$ such that $h^{s_{a}}(a) \in$ $A^{*} L^{r_{a}} w_{a}, h^{s_{b}}(b) \in w_{b} R^{r_{b}} A^{*}$, where $w_{a}, w_{b} \in M_{F}^{*}$.

Let $N_{a b}=$ l.c.m. $\left(r_{a}\right.$, l.m. $\left.\left(w_{a} u w_{b}\right), r_{b}\right)$. For any $n \in\left[0, N_{a b}-1\right]$ it holds

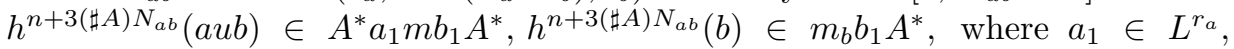
$b_{1} \in R^{r_{b}}, m, m_{b} \in\left(M \cup M_{R}\right)^{*}$ and $m_{b} b_{1}$ is a suffix of $m b_{1}$. Let $p=\left|a_{1} m b_{1}\right|$. Now we are ready to formulate

Lemma 4.17. Let $a u b \in L R, a, b \in A$. For an arbitrary (but fixed) $n \in\left[0, N_{a b}-1\right]$ we denote $w=h^{n+3(\sharp A) N_{a b}}(a u b)$ and $\left(b_{1}, i\right)=$ first $_{E, \mathbb{Z}}\left(h^{n+3(\sharp A) N_{a b}}(b)\right)$. Let $k \in \mathbb{N}$ point out the position of the letter $b_{1}$ in the word $w$, that is, $k=\left|h^{n+3(\sharp A) N_{a b}}(a u)\right|$ $+i$. Finally let $j \in \mathbb{N}$ point out the first position of the letter $b_{1}$ in $h^{N_{a b}}\left(b_{1}\right)$.

1. $N_{a b}$ is the smallest integer such that $\left(h^{N_{a b}}\right)^{*, j}\left(w^{k}\right)=\left(h^{N_{a b}}\right)^{*, j}\left(\left(a_{1} m b_{1}\right)^{(p)}\right) \in$ $\delta L_{h}$ and the equivalence class of $\left(h^{N_{a b}}\right)^{*, j}\left(w^{k}\right)$ is in $\delta L_{h} / \sigma^{*}$.

2. If $z$ is a primitive word then the following conditions are equivalent:

(i) $\left(h^{N_{a b}}\right)^{*, j}\left(\left(a_{1} m b_{1}\right)^{(p)}\right) \in{ }^{\omega} z z z^{\omega}$;

(ii) there exists a number $r \in[1, \sharp A]$ and the word $v \in P^{r}$ such that $a_{1} m b_{1}$ is a factor of $v v$ and $v \approx z$.

3. It is decidable, whether or not the equivalence classes of the periodic words characterized in 1 are equal.

Proof. From Lemmas 3.4 and 3.5 and the construction of the word $\left(h^{N_{a b}}\right)^{*, j}\left(w^{k}\right)$, repeating the argumentation from Lemmas 4.1, 4.9 and 4.11 we prove the statement 1 .

Now assume 2(i). Lemma 4.1 and the fact that $b_{1} \in R^{r_{b}}$ imply that $\left(h^{N_{a b}}\right)^{*, j}\left(b_{1}\right)=x z z^{\omega}$ where $x$ is a suffix of $z$. Similarly $\left(h^{r_{b}}\right)^{*, j}\left(b_{1}\right)=x z z^{\omega}$. Hence there exists a primitive word $v$ such that $v \approx z$ and $x z$ is a prefix of $v v$ and $v \in P^{r_{b}}$. The word $a_{1} m b_{1}$ is a factor of $v v$ and in consequence 2(ii) follows from 2(i).

Assume now 2(ii). Taking into account the fact that $b_{1} \in R^{r_{b}}$ and on the basis of Lemma 4.15 we obtain $\left(h^{r_{b}}\right)^{*, j}\left(b_{1}\right)=y v v^{\omega}$ where $y$ is a suffix of $v$. Hence there exists a word $u$ such that $u \approx v$ and $y v$ is a prefix of $u u$. It is straightforward to observe that $\left(h^{r_{b}}\right)^{*, j}\left(b_{1}\right)=u u^{\omega}$. From the description of $a_{1} m b_{1}$ we conclude that $a_{1} m b_{1}$ is a factor of $u u$.

According to Lemma 4.14 it follows that $h^{r_{b}}(u) \in u^{+} u$. Since $r_{b}$ is a divisor of $N_{a b}$ we conclude that the word $h^{i N_{a b}}\left(a_{1} m b_{1}\right)$ is a factor of $h^{i N_{a b}}(u u) \in u^{+} u$ for $i \in \mathbb{N}$. Hence the equality $\left(h^{N_{a b}}\right)^{*, j}\left(\left(a_{1} m b_{1}\right)^{(p)}\right)={ }^{\omega} u u u^{\omega}$ follows directly from the 
statement 1 . Just from the assumptions $u \approx z$, and there exists a word $z=v_{1} a v_{2}$ such that $\left(h^{N_{a b}}\right)^{*, j}\left(\left(a_{1} m b_{1}\right)^{(p)}\right) \in \omega^{\omega} z z z^{\omega}$.

The statement 3 follows according to the fact that two periodic words are in relation if and only if their primitive periods are in relation.

This completes the proof of the lemma.

Lemma 4.18. Let $a u b \in L R, a, b \in A$. For an arbitrary (but fixed) $n \in\left[0, N_{a b}-1\right]$ we denote $w=h^{n+3(\sharp A) N_{a b}}(a u b)$ and $\left(b_{1}, i\right)=$ first $_{E, \mathbb{Z}}\left(h^{n+3(\sharp A) N_{a b}}(b)\right)$. Let $k \in \mathbb{N}$ point out the position of the letter $b_{1}$ in $w$, that is, $k=\left|h^{n+3(\sharp A) N_{a b}}(a u)\right|+i$. Finally let $j \in \mathbb{N}$ point out the first position of the letter $b_{1}$ in $h^{N_{a b}}\left(b_{1}\right)$. For $c v d \in L R, c, d \in A$ we denote $\bar{w}=h^{m+3(\sharp A) N_{c d}}(c v d)$ and let $r$ be the counterpart of $k, p$ of $j$.

The following statements are true:

(i) $\left\{\left(h^{N_{a b}}\right)^{*, j}\left(w^{(k)}\right)\right\}_{n \in \mathbb{N}}$ is a set of $N_{a b}$ words in $\delta L_{h}$;

(ii) it is decidable, whether or not the words $\left(h^{N_{a b}}\right)^{*, j}\left(w^{(k)}\right)$ and $\left(h^{N_{c d}}\right)^{*, p}\left(\bar{w}^{(r)}\right)$ are equal;

(iii) if $\left(h^{N_{a b}}\right)^{*, j}\left(w^{(k)}\right),\left(h^{N_{c d}}\right)^{*, p}\left(\bar{w}^{(r)}\right)$ are two different words then their equivalence classes are equal if and only if there exist $s \in[1, \sharp A]$ and $z \in P^{r}$ such that

$$
\left(h^{N_{a b}}\right)^{*, j}\left(w^{(k)}\right),\left(h^{N_{c d}}\right)^{*, p}\left(\bar{w}^{(r)}\right) \in{ }^{\omega} z z z^{\omega} .
$$

Proof. The statement (i) follows directly from Lemma 4.17.

Now observe that

$$
\left(h^{N_{a b}}\right)^{*, j}\left(w^{(k)}\right)=w_{L}\left(a_{1} m b_{1}\right)^{\left(e_{1}\right)} w_{R} \quad \text { where } \quad e_{1}=\left|a_{1} m b_{1}\right|
$$

and

$$
\left(h^{N_{c d}}\right)^{*, p}\left(\bar{w}^{(r)}\right)=v_{L}\left(c_{1} n d_{1}\right)^{\left(e_{2}\right)} v_{R} \quad \text { where } \quad e_{2}=\left|c_{1} n d_{1}\right|
$$

for some one-sided infinite words $w_{L}, w_{R}, v_{L}, v_{R}$, finite words $m, n$ and letters $a_{1}, b_{1}, c_{1}, d_{1}$.

From Lemma 3.3 and the proof of Lemma 4.17 the equality $w_{L}\left(a_{1} m b_{1}\right)^{\left(e_{1}\right)} w_{R}=$ $v_{L}\left(c_{1} n d_{1}\right)^{\left(e_{2}\right)} v_{R}$ holds if and only if $a_{1} m b_{1}=c_{1} n d_{1}$ which proves (ii).

For the proof of (iii) let us assume that words $\left(h^{N_{a b}}\right)^{*, j}\left(w^{(k)}\right)$ and $\left(h^{N_{c d}}\right)^{*, p}\left(\bar{w}^{(r)}\right)$ are equal modulo the origin. First consider the case:

$$
(*)\left\{\begin{array}{l}
\left(h^{N_{a b}}\right)^{*, j}\left(w^{(k)}\right)=w_{L}\left(a_{1} m b_{1}\right)^{\left(e_{1}\right)} w_{0} c_{1} n d_{1} v_{R} \\
\left(h^{N_{c d}}\right)^{*, p}\left(\bar{w}^{(r)}\right)=w_{L} a_{1} m b_{1} w_{0}\left(c_{1} n d_{1}\right)^{\left(e_{2}\right)} v_{R}
\end{array}\right.
$$

where $w_{0} \in A^{*}$. 
If $K=$ l.c.m. $\left(N_{a b}, N_{c d}\right)$, then there exists the smallest positive integer $t \in$ $\mathbb{N} \backslash\{0\}$ such that

$$
\begin{aligned}
\left(h^{t K}\right)^{j}\left(\left(a_{1} m b_{1}\right)^{\left(e_{1}\right)}\right) & =\ldots\left(a_{1} m b_{1}\right)^{\left(e_{1}\right)} w_{0} c_{1} n d_{1} \ldots \\
\left(h^{t K}\right)^{p}\left(\left(c_{1} n d_{1}\right)^{\left(e_{2}\right)}\right) & =\ldots a_{1} m b_{1} w_{0}\left(c_{1} n d_{1}\right)^{\left(e_{2}\right)} \ldots
\end{aligned}
$$

Since $h^{t K}(v)=\ldots v w_{1} v \ldots$ then for $k \geq 1$ we have

$$
(* *) \quad\left(h^{t K}\right)^{k+1}(v)=\ldots v u_{k} v w_{k+1} v u_{k} v \ldots
$$

where $u_{1}=w_{1}, u_{i+1}=u_{i} v w_{i+1} v u_{i}$ for $i \in\{1,2, \ldots, k-1\}$.

For every finite factor of $\left(h^{t K}\right)^{*}(v)$ considered as a finite word with the origin fixed on $b_{1}$ or $d_{1}$ there exists the number $s \in \mathbb{N} \backslash\{0\}$ such that this word is a factor of $\left(h^{t K}\right)^{s}(v)$ with the origin fixed on $b_{1}$ or $d_{1}$ respectively (see Lem. 4.17). Hence from Lemma 4.17 and the condition $(*)$ above we have

$$
\begin{aligned}
\left(h^{t K}\right)^{*, j}\left(v w_{1} v^{(k)}\right) & =w_{L} v^{(k)} v_{R}, \\
\left(h^{t K}\right)^{*, j}\left(v^{(k)}\right) & =w_{L} v^{(k)} v_{R} \\
\left(h^{t K}\right)^{*, p}\left(v^{(r)}\right) & =w_{L} v^{(r)} v_{R} .
\end{aligned}
$$

Since $h^{t K}\left(v^{(k)}\right)=\ldots v^{(k)} w_{1} v \ldots,\left(h^{t K}\right)^{*, p}\left(v^{(r)}\right)=\ldots v w_{1} v^{(r)} \ldots$ then by induction on $k$ we obtain

$$
\begin{array}{rlr}
\left(h^{t K}\right)^{k}\left(v w_{1} v^{(k)}\right) & =\ldots v u_{k} v w_{k+1} v^{(k)} u_{k} \ldots \\
\left(h^{t K}\right)^{k+1}\left(v^{(k)}\right) & = & \ldots v^{(k)} u_{k} v w_{k+1} v u_{k} v \ldots \\
\left(h^{t K}\right)^{k+1}\left(v^{(r)}\right) & =\ldots v u_{k} v w_{k+1} v u_{k} v^{(r)} u_{k} \ldots
\end{array}
$$

From the above, Lemmas 3.4 and 3.5 we conclude that for any $i \in\{1,2, \ldots, k\}$ words $v u_{i} v w_{i+1} v^{(k)} u_{i}, v^{(k)} u_{i} v w_{i+1} v u_{i} v$ are factors of $\left(h^{t K}\right)^{*, j}\left(v^{(k)}\right)$ and $v u_{i} v w_{i+1} v u_{i} v^{(r)}$ is a factor of $\left(h^{t K}\right)^{*, p}\left(v^{(r)}\right)$.

Hence for $k \geq 1$ and every $i \in\{1,2, \ldots, k\}$

$$
(* * *) \quad u_{i} v w_{i+1}=w_{i+1} v u_{i}
$$

For every $k \geq 1$ there exist $n_{k} \in \mathbb{N} \backslash\{0\}$ such that

$$
\left(h^{t K}\right)^{n_{k}}\left(v^{(r)}\right)=\ldots v u_{k} v w_{k+1} v u_{k} v^{(r)} u_{k} v w_{k+1} v u_{k} v \ldots
$$


The above observations and properties $(* *),(* * *)$ imply that for every $k \geq 1$

$$
\begin{aligned}
& \left(h^{t K}\right)^{n_{k}}\left(v^{(r)}\right)= \\
& \quad \ldots v w_{k+1}\left(v w_{k}\right)^{2}\left(v w_{k-1}\right)^{2^{2}} \ldots\left(v w_{1}\right)^{2^{k}} v^{(r)}\left(w_{1} v\right)^{2^{k}} \ldots\left(w_{k-1} v\right)^{2^{2}}\left(w_{k} v\right)^{2} w_{k+1} v \ldots
\end{aligned}
$$

Hence

$$
\left(h^{t K}\right)^{*, p}\left(v^{(r)}\right)={ }^{\omega}\left(v w_{1}\right) v^{\sigma(r)}\left(w_{1} v\right)^{\omega}
$$

and

$$
\begin{aligned}
& \left(h^{N_{c d}}\right)^{*, p}\left(\bar{w}^{(r)}\right)=\left(h^{N_{c d}}\right)^{*, p}\left(v^{(r)}\right)={ }^{\omega}\left(v w_{1}\right) v^{(r)} w_{1}\left(v w_{1}\right)^{\omega} \\
& \left(h^{N_{a b}}\right)^{*, j}\left(w^{(k)}\right)=w_{L} v^{(k)} v_{R}=^{\omega}\left(v w_{1}\right) v^{(k)} w_{1}\left(v w_{1}\right)^{\omega} .
\end{aligned}
$$

Thus there exists a primitive word $z_{p}$ such that $v w_{1} \in z_{p}^{+}$. By Lemma 4.17 there exist a number $r \in[1, \sharp A]$ and a word $z \in P^{r}$ such that $z \approx z_{p}$. It is not difficult to observe that the similar proof works when we drop the assumption $(*)$.

Example 4.19. We have $L R=\left\{a^{\prime} e a\right\}, N_{a^{\prime} a}=2$. For $n=0,1$ there are two words $w=h^{0+3 \cdot 8 \cdot 2}\left(a^{\prime} e a\right)=e^{48} a^{\prime} e a e^{48}$ and $\bar{w}=h^{1+3 \cdot 8 \cdot 2}\left(a^{\prime} e a\right)=e^{49} b^{\prime} e b e^{49}$ for which first $_{E, \mathbb{Z}}\left(h^{48}(a)\right)=(a, 0)$ and first $_{E, \mathbb{Z}}\left(h^{49}(a)\right)=(b, 0)$ respectively, $k=50$ and $j=0$ points out the first position of $a$ in $h^{2}(a)$ and the first position of $b$ in $h^{2}(b)$. Lemma 4.18 implies that $\left(h^{2}\right)^{*, 0}\left(w^{(50)}\right)={ }^{\omega} e a^{\prime} e a e^{\omega}$ and $\left(h^{2}\right)^{*, 0}\left(\bar{w}^{(50)}\right)={ }^{\omega} e b^{\prime} e b e^{\omega}$ are in $\delta L_{h}$.

\section{MAIN RESUlT}

Now we are able to formulate and prove the main result of our paper characterizing pasted words, that is, words in $\delta L_{h}$. Remind that the set of pasted words is a subset of the boundary set $\partial L_{h}$ and both are in the metric space $(\Omega, d)$. In presented below theorem we consider equivalence classes of pasted words so we work in $\Omega / \sigma^{*}$. In the sequel $[w]$ denotes an equivalence class of a word $w \in \Omega$.

Theorem 5.1. Let $h: A^{*} \rightarrow A^{*}$ be a morphism.

1. For any equivalence class $[w] \in \delta L_{h} / \sigma^{*}$ there exists a representant of the class such that it is a fixed point of $h^{N}$ for some effectively computable integer $N$ and is constructable as an infinite iterate of $h^{N}$ on some letter in $A$.

2. It is decidable whether or not the equivalence classes $[w]$ and $[v]$ in $\delta L_{h} / \sigma^{*}$ are equal.

3. The set $\delta L_{h} / \sigma^{*}$ is finite. Hence there exists an algorithm describing effectively (in the sense of 1 ) the set $\delta L_{h} / \sigma^{*}$. 
Proof. Assume first that $w \in \partial L_{h}$ is right-infinite and that a sequence $\left\{z_{i}\right\}_{i \in \mathbb{N}}$ of words of $L_{h}$ converges to $w$. Now there are two possibilities:

(i) $\operatorname{first}_{E}(w)=\emptyset$, then $w=\operatorname{mau}_{1}$, where $m \in M^{*}$ and $\operatorname{first}_{M_{R}, \mathbb{Z}}(w)=(a, e)$. In this case denote $w_{a}=\left(\operatorname{mau}_{1}\right)^{(e)}$. Notice that $w_{a} \in[w]$;

(ii) $\operatorname{first}_{E, \mathbb{Z}}(w)=(b, f)$ then $w=m b u_{2}$, where $m \in\left(M \cup M_{R}\right)^{*}$. In this case denote $w_{b}=\left(m b u_{2}\right)^{(f)}$. Notice that $w_{b} \in[w]$.

For $s \in\{a, b\}$ one can choose from $\left\{\left[z_{i}\right]\right\}_{i \in \mathbb{N}}$ a sequence $\left\{z_{i}^{s}\right\}_{i \in \mathbb{N}}$ of words with origins which converges to $w_{s}$. Hence there exists for $s \in\{a, b\}$ a subsequence $\left\{z_{i_{k}}^{s}\right\}_{k \in \mathbb{N}}$ such that $z_{i_{k}}^{s} \in h^{n+n_{k} r}(c)$, where $c \in E \cup I_{E}, n_{k} \in \mathbb{N}$, and numbers $n \in \mathbb{N}$, $r \in[1, \sharp A]$ are the smallest ones such that first $E, \mathbb{Z} h^{n+j r}(c)=$ first $_{E, \mathbb{Z}} h^{n+(j+1) r}(c)$ for each $j \in \mathbb{N}$. Now Lemmas 4.1 and 4.5 imply that there exists the smallest integer $p \in \mathbb{N} \backslash\{0\}$ such that the sequence $y_{k}^{s}=h^{n+k \cdot p \cdot r}(c)$ converges to $w_{s}$ in both of the above cases. Again from Lemmas 4.1 and 4.5 it follows that there exists an integer $N$, such that $N$ is a divisor of $p \cdot r$ and $w_{s}$ is a fixed point of $h^{N}$. That is $w_{a}=\left(h^{N}\right)^{*, \bar{e}}\left(w_{a}\right)$ where $\bar{e}=|h(a)|-1$ and similarly for $w_{b}$.

By symmetry we obtain the same result for left-infinite word $w \in \partial L_{h}$. This lead us to the conclusion that all one-sided infinite words in $\partial L_{h}$ (and in $\delta L_{h}$ ) are described by Lemmas 4.1 and 4.5 and imply for this type of words the statement 1 of the theorem.

Now assume that $w \in \partial L_{h}$ is a pasted word. Hence $w=u m v$ where $m \in$ $\left(M \cup M_{R}\right)^{*}$ and $u, v \in \partial L_{h}$ are left and right infinite, respectively. It means that $h^{N_{1}}\left(u_{s}\right)=u_{s}$ and $h^{N_{2}}\left(v_{r}\right)=v_{r}$ for some $u_{s}$ a word in $[u]$ such that $s$ is the origin of $u$ and $v_{r}$ in $[v]$ with $r$ as the origin and for some positive integers $N_{1}, N_{2}$. According to Lemma 3.4 there exists the smallest integer $N \in \mathbb{N} \backslash\{0\}$ such that $h^{N}(m)=m$ and puting l.c. $m\left(N_{1}, N_{2}, N\right)=L$ we have:

(i) $u m v$ is a fixed point of $h^{L}$ if the origin of $u m v$ is equal $r \in E$ and if $s \in M_{R}$ (Lem. 4.11);

(ii) $u m v$ is a fixed point of $h^{L}$ if the origin of $u m v$ is equal $s$ in all other cases.

Thus all pasted words in $\partial L_{h}$ are described by Lemmas 4.9, 4.11, 4.17 and 4.18, what finishes the proof of the statement 1 .

The statement 2 and 3 follows directly from Lemmas 3.1, 3.3, 4.1, 4.5, 4.9, 4.11, 4.14 and $4.17,4.18$ what finishes the proof of the theorem.

Example 5.2. Taking for example an equivalence class $\left[{ }^{\omega} e b^{\prime} e b e^{\omega}\right] \in \delta L_{h} / \sigma^{*}$ and ${ }^{\omega} e b^{\prime} e b e^{\omega} \in\left[{ }^{\omega} e b^{\prime} e b e^{\omega}\right]$ such that the origin is set on the $e$ between $b^{\prime}$ and $b$ observe that $h^{2}\left({ }^{\omega} e b^{\prime} e b e^{\omega}\right)={ }^{\omega} e b^{\prime} e b e^{\omega}$ and $\left(h^{2}\right)^{*, 0}\left(b^{\prime} e b^{(2)}\right)={ }^{\omega} e b^{\prime} e b e^{\omega}$. On the base of all examples we have $\delta L_{h}$ equals

$$
\left\{a e^{\omega}, b e^{\omega},{ }^{\omega} e a^{\prime},{ }^{\omega} e b^{\prime}, e e^{\omega},{ }^{\omega} e e,{ }^{\omega} e e e^{\omega},{ }^{\omega} e a e^{\omega},{ }^{\omega} e b e^{\omega},{ }^{\omega} e a^{\prime} e a e^{\omega},{ }^{\omega} e b^{\prime} e b e^{\omega}\right\} .
$$

Acknowledgements. I wish to thank the referees for their really valuable suggestions and comments which significantly helped to clarify the presentation of this paper. 


\section{REFERENCES}

[1] A. Ehrenfeucht and G. Rozenberg, Simplifications of homomorphism. Inform. Control 38 (1978) 298-309.

[2] W. Foryś and T. Head, The poset of retracts of a free monoid. Int. J. Comput. Math. 37 (1990) 45-48.

[3] T. Harju and M. Linna, On the periodicity of morphism on free monoid. RAIRO: Theoret. Informatics Appl. 20 (1986) 47-54.

[4] T. Head, Expanded subalphabets in the theories of languages and semigroups. Int. J. Comput. Math. 12 (1982) 113-123.

[5] T. Head and V. Lando, Fixed and stationary $\omega$-wors and $\omega$-languages. The book of L, Springer-Verlag, Berlin (1986) 147-155.

[6] M. Lothaire, Combinatorics on words. Addison-Wesley (1983).

[7] J. Matyja, Sets of primitive words given by fixed points of mappings. Int. J. Comput. Math. (to appear).

[8] P. Narbel, Limits and boundaries of words and tiling substitutions. LITP, TH93.12 (1993).

[9] P. Narbel, The boundary of iterated morphisms on free semi-groups. Int. J. Algebra Comput. 6 (1996) 229-260.

[10] J. Shallit and M. Wang, On two-sided infinite fixed points of morphisms. Lect. Notes Comput. Sci. 1684 (1999) 488-499.

Communicated by A. Restivo.

Received May 5, 2002. Accepted November 7, 2003.

To access this journal online: www.edpsciences.org 